\title{
Implementation of DWT using Haar Transform with Felics Algorithm
}

\author{
Sowmyashree M S ${ }^{1}$, Saritha I G ${ }^{2}$,Naveen I G ${ }^{3}$ \\ Assistant Professor, Department of Telecommunication Engineering, BMSIT ${ }^{1,2}$ \\ Assistant Professor, Department of Electronics and communication Engineering, Sir MVIT ${ }^{3}$
}

\begin{abstract}
This presents an approach towards implementation of the Discrete Wavelet Transform (DWT) for image compression. The design follows a specific standard and can be used for lossless compression. Wavelets are mathematical functions that cut up data into different frequency components, and then study each component with a resolution matched to its scale. The demand for obtaining a higher quality images and transmitting them over the internet has created a strong need to develop algorithms and coding for such images. The algorithm included in this paper is the Felics algorithm which includes adjusted binary code \& golomb rice coding. Transformation used is the Haar transform since it is advantageous over other transforms.
\end{abstract}

Index Terms: Wavelet, Haar, Felics, MSE, CR, PSNR, Elapsed time.

\section{INTRODUCTION}

Image compression is a fast paced and dynamically The basic objective of image compression is to find an changing field with many different varieties of image representation in which pixels are less correlated. compression methods available. Images contain large The two fundamental principles used in image amount of data hidden in them, which is highly correlated. compression are redundancy and irrelevancy. Redundancy A common characteristic of most images is that the removes redundancy from the signal source and neighboring pixels are correlated and therefore contain irrelevancy omits pixel values which are not noticeable by redundant information.

Need for image compression:

- To save memory required to store the image

- To save transmission band width

- To save transmission time

With the use of digital cameras, requirements for storage, manipulation, and transfer of digital images, has grown explosively. These image files can be very large and can occupy a lot of memory. Image data comprise of a significant portion of the multimedia data and they occupy the major portion of the communication bandwidth for multimedia communication. Therefore development of efficient techniques for image compression has become quite necessary.

Image compression is the process of reducing the amount of data required to represent an image. Image compression is done to reduce irrelevance and redundancy of the image data in order to be able to store or transmit data in an efficient form. Uncompressed images require considerable storage capacity and transmission bandwidth.

The objective of image compression technique is to reduce redundancy of the image data in order to be able to store or transmit data in an efficient form. This results in the reduction of file size and allows more images to be stored in a given amount of disk or memory space Image compression may be lossy or lossless. Lossless compression is preferred for archival purposes and often for medical imaging, technical drawings, clip art, or comics. In a lossless compression algorithm, compressed data can be used to recreate an exact replica of the original; no information is lost to the compression process human eye.

There are a number of problems to be solved in image compression to make the process viable and more efficient. Design of computationally efficient and effective software algorithms for lossless image compression forms the primary objective. ${ }^{[1]}$

The Two-Dimensional DWT converts images from spatial domain to frequency domain. It can be accomplished by applying one-dimensional filter banks in a separable manner. At each level of the wavelet decomposition, each row of an image is first transformed using a $1 \mathrm{D}$ vertical analysis filter-bank. The same filter-bank is then applied horizontally to each row of the filtered and sub sampled data.

One-level of wavelet decomposition produces four filtered and sub sampled images, referred to as sub bands. The four sub bands are denoted as horizontally and vertically low pass (LL1), horizontally high pass and vertically low pass (HL1), horizontally low pass and vertically high pass (LH1), and horizontally high pass and vertically high pass (HH1). The bands other than LL1 generally have small values. ${ }^{[2]}$

In addition to the above properties of wavelet transform, The Haar wavelet transformation is composed of a sequence of low-pass and high-pass filters, known as a filter bank.

The low pass filter per-forms an averaging/blurring operation and the high-pass filter performs a differencing operation.

The result of the complete transformation, $\mathrm{T}$, is composed of 4 new sub-images, which correspond to the blurred image and the vertical, diagonal, and horizontal 
differences between the original image and the blurred image.

The blurred representation of the image removes the details (high frequency components), which are represented separately in the other three images, in a manner that produces a sparser representation overall, making it is easier to store and transmit. ${ }^{[3]}$

The Haar system is an orthonormal system on the interval $[0,1]$. The Haar wavelet is a first known wavelet which has an orthonormal basis. It is a sequence of functions supported on the small subintervals of length $[0,1]$ : The Haar basis functions are step functions with jump discontinuities. The Haar wavelet transform is used to compress one and two dimensional signals. ${ }^{[4]}$

\section{II.DISCRETE WAVELET TRANSFORM}

The Discrete Wavelet Transform, which is based on subband coding, is found to yield a fast computation of Wavelet Transform. It is easy to implement and reduces the computation time and resources required. DWT adopt fast algorithm of two dimensional wavelet transform. The original image is decomposed into four sub-bands after passing a high- pass filter and low- pass filter. The combination of high pass $\mathrm{g}[\mathrm{n}]$ and low pass filter $\mathrm{h}[\mathrm{n}]$ comprise a pair of analyzing filters. The output of each filter contains half the frequency content, but an equal amount of samples as the input signal.

The four sub-bands are LL, HL, LH and HH respectively. $\mathrm{LL}$ is a low frequency sub-band of the approximate image. $\mathrm{HL}$ is a high frequency sub-band of the horizontal details of the image. LH is a high frequency sub-band of the vertical details of the image. $\mathrm{HH}$ is a high frequency sub-band of the diagonal details of the image. The process is called the first level of wavelet decomposition. The low frequency sub-band can be continually decomposed into four subbands.

\begin{tabular}{|c|c|c|c|c|c|c|c|}
\hline \multirow[t]{2}{*}{ LL } & \multirow[t]{2}{*}{ HL } & LL & HL & \multirow[t]{2}{*}{ HL } & \begin{tabular}{|l|l|}
$\mathrm{LI}$ & $\mathrm{HL}$ \\
$\mathrm{LH}$ & $\mathrm{HH}$ \\
\end{tabular} & HL & \multirow[t]{2}{*}{ HL } \\
\hline & & LH & $\mathrm{HH}$ & & LH & $\mathrm{HH}$ & \\
\hline LH & $\mathrm{HH}$ & & & $\mathrm{HH}$ & $\mathrm{Ll}$ & & $\mathrm{HH}$ \\
\hline
\end{tabular}

Level of Wavelet decomposition

Discrete Wavelet Transform (DWT) has emerged as a popular Technique for image compression applications with excellent compression performance. The WT requires much less hardware to implement than Fourier methods, such as the DCT. One of the shortcomings of using DCT is blocking artifact and bad subjective quality when the images are restored by this method at the high compression ratios. Therefore, much of the research activities in image compression have been focused on the discrete wavelet transform. WT of an image represents image as a sum of wavelets on multi-resolution levels. In wavelet transform any one-dimensional function is transformed into a twodimensional space, where it is approximated by coefficients that depend on time and on scale. Hence the wavelet transform is well suited for image compression. The type of wavelet transform will directly impact on of the complexity of the calculation and, indirectly, affect the image compression and reconstruct the image with an acceptable error

\section{HAAR TRANSFORM}

The Haar transform is simple, efficient in memory usage due to high zero value spread (it can use sparse principle), and exactly reversible without the edge effects as compared to DCT (Discrete Cosine Transform). The implemented Matlab simulation results prove the effectiveness of DWT (Discrete Wave Transform) algorithms based on Haar techniques in attaining an efficient compression ratio (C.R), achieving higher peak signal to noise ratio (PSNR), and the resulting images are of much smoother as compared to standard JPEG especially for high C.R.

A comparison between Haar, Daubecheis, Symlet, Biorthogonal techniques is done finally which approves the highest capability of Haar between others.

The Haar wavelet transformation is composed of a sequence of low-pass and high-pass filters, known as a filter bank.

\section{Properties of Haar wavelet transform:}

1. Haar Transform is real and orthogonal. Therefore
1. $\mathrm{Hr}=\mathrm{Hr}^{*}$
2. $\mathrm{Hr}=\mathrm{Hr}$

Haar Transform is a very fast transform.

2. The basis vectors of the Haar matrix are sequence ordered.

3. Haar Transform has poor energy compaction for images.

4. Orthogonality: The original signal is split into a low and a high frequency part and filters enabling the splitting without duplicating information are said to be orthogonal.

5. Linear Phase: To obtain linear phase, symmetric filters would have to be used.

6. Compact support: The magnitude response of the filter should be exactly zero outside the frequency range covered by the transform. If this property is algebra satisfied, the transform is energy invariant.

7. Perfect reconstruction: If the input signal is transformed and inversely transformed using a set of weighted basis functions and the reproduced sample values are identical to those of the input signal, the transform is said to have the perfect reconstruction property. If, in addition no information redundancy is present in the sampled signal, the wavelet transform is, as stated above, orthonormal.

\section{Advantages of Haar Wavelet transform}

1. Best performance in terms of computation time.

2. Computation speed is high.

3. Lossless compression.

4. Simplicity (e.g., if, =0).

5. HWT is efficient compression method.

6. It is memory efficient, since it can be calculated in place without a temporary array 


\section{IV.ALGORITHM FOR IMAGE COMPRESSION USING DWT}

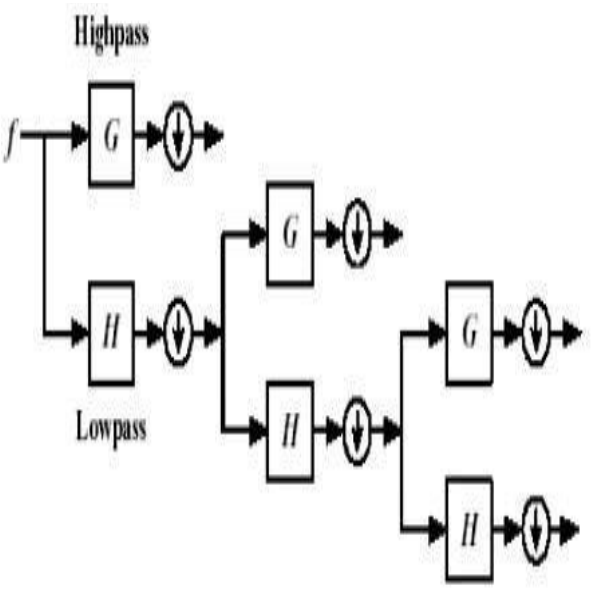

Multilevel Decomposition using low pass and high pass filters for image compression using wavelets

Algorithm uses a quantization approach that divides the input image in 4 filter coefficients and then performs further quantization on the lower order filter. This quantization is depending upon the decomposition levels and maximum numbers of decomposition levels to be entered are 3 for DWT. DWT exploits the interpixel redundancies in order to render excellent decorrelation for most natural images. Thus the uncorrelated transform coefficients can be encoded independently without compromising coding efficiency. DWT packs energy in the low frequency regions.

Therefore, some of the high frequency content can be discarded without significant quality degradation. Such a quantization scheme causes further reduction in the entropy or average number of bits per pixel.

The attributes of the DWT have led to its widespread deployment in virtually every image/video processing standard of the last decade, for example, JPEG (classical), MPEG- 1, MPEG-2, MPEG-4, and MPEG-4 FGS. Nevertheless, the DWT still offers new research directions that are being explored in the current and upcoming image/video coding standards.

\section{PERFORMANCE CRITERION}

The performance is evaluated by the following parameters: 1. Distortion measure

Mean Square Error (MSE) is used to measure the rate of distortion in the reconstructed image. Mean square error (MSE) is widely used to measure the degree of image distortion because they can represent the overall gray-value error contained in the entire image and are mathematically tractable as well.

$$
M S E=\frac{1}{m n} \sum_{i=0}^{m-1} \sum_{j=0}^{n-1}[I(i, j)-K(i, j)]^{2}
$$

\section{PSNR}

PSNR is used as an approximation to human perception of reconstruction quality. PSNR has been accepted as a widely used quality measurement in the field of image compression.

$$
\begin{aligned}
\text { PSNR } & =10 \cdot \log _{10}\left(\frac{\mathrm{MAX}_{1}^{2}}{\mathrm{MSE}}\right) \\
& =20 \cdot \log _{20}\left(\frac{\mathrm{MAX}_{1}}{\sqrt{\mathrm{MSE}}}\right)
\end{aligned}
$$

\section{Compression Ratio}

The Compression ratio (CR) is the ratio between the size of the original image and the size of the compressed image. Compression Ratio $=$ Original image size/ Compressed image size.

It Compare sizes of original and compressed images and ratio of sizes tells us more about effectiveness. Compression ratio is defined as the ratio of the number of nonzero elements in original matrix to the number of nonzero elements in updated transformed matrix.

The Compression Ratio is at threshold parameter that is it indicates the extent up to which the input image can be compressed beyond which the original image loses its effectiveness

\section{Elapsed time for compression}

The time taken to compress the original image or time taken to decompress the compressed image is called Elapsed time for compression.

This time is dependent on various parameters like the complexity of the algorithm used to compress and decompress the image, the processor which is performing the same and the size of the image being used.

The time taken varies from processor to processor and is considered to have less value in more sophisticated processors than normal processors. The processing speed plays a vital role in determining the time elapsed.

\section{FELICS ALGORITHM}

In this algorithm raster-scan order is used, and a pixel's two nearest neighbours are used to directly obtain an approximate probability distribution for its intensity, in effect combining the prediction and error modelling steps. [11]

FELICS utilizes two reference pixels around current pixel to yield the prediction template, and it can be divided into four cases. In case 1 , since surrounding reference pixels are not available for the first two pixels, P1 and P2, both current pixels are directly packed into bit stream with original pixel intensity. For case 2, successive pixels, N1 and N2, are regarded as reference pixels for current pixel P5. For non-first row, cases 3 and 4 clearly define the relationship between current pixel and reference pixels. Between $\mathrm{N} 1$ and $\mathrm{N} 2$, the smaller reference pixel is represented as L, and the other one is $\mathrm{H}$. As in Figure shown below, the intensity distribution model is exploited to predict the correlation between current pixel and reference pixels. In this model, the intensity that occurs between $\mathrm{L}$ and $\mathrm{H}$ is with almost uniform distribution, and regarded as in-range. The intensity higher than $\mathrm{H}$ value or smaller than $\mathrm{L}$ value is considered as above range and below range, respectively. For in-range, the adjusted binary code is adopted, and Golomb-Rice code is for both above range and below range 


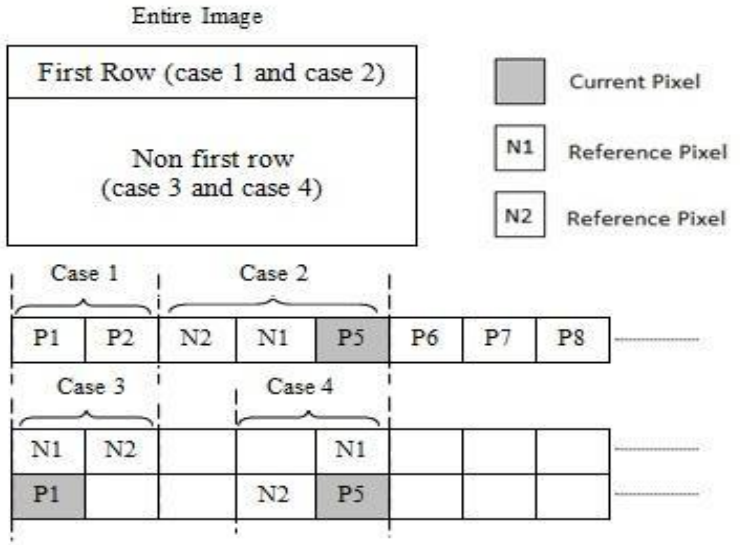

Adjusted Binary Code:

The adjusted binary code is adopted in in-range, where the intensity of current pixel is between $\mathrm{H}$ and $\mathrm{L}$. For in-range, the probability distribution is slightly higher in the middle section and lower in both side sections. Therefore, the shorter codeword is assigned to the middle section, and longer one is assigned to both side sections. To describe the coding flow of adjusted binary code, the coding parameters should be first computed as follows:

Delta $=\mathrm{H}-\mathrm{L}$

Range $=$ delta +1

Upper bound $=$ ceil $\log 2$ (range)

Lower bound $=$ floor $\log 2$ (range) threshold $=$ 2upper_bound - range shift number $=($ range - threshold $) /$ 2

The adjusted binary code takes the sample of $\mathrm{P}-\mathrm{L}$ to be encoded, and range indicates that the number of possible samples should be encoded for a given delta. The upper bound and lower bound denote the maximum and minimum number of bits to represent the codeword for each sample, respectively. Particularly, the lower bound is identical to upper bound, while the range is exactly equal to the power of two. The threshold and shift number are utilized to determine which sample should be encoded with upper bound bit or lower bound bit.

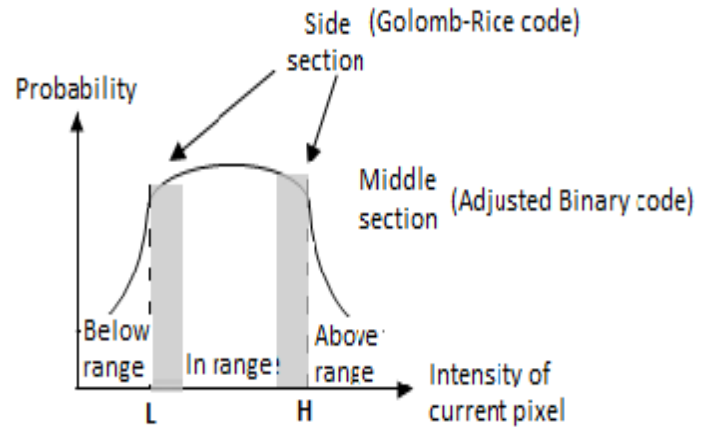

Probability distribution model in FELICS

Golomb-Rice Code:

For both above range and below range, the probability distribution sharply varies with exponential decay rate, and the efficient codeword should be more intensively assigned to the intensity with high probability. Therefore, Golomb-Rice code is adopted as the coding tool for both above range and below range. With Golomb-Rice code, the codeword of sample $\mathrm{x}$ is partitioned into unary and binary parts:

Unary part: floor $(\mathrm{x} / 2 \mathrm{k})$ Binary part: $\mathrm{x} \bmod 2 \mathrm{k}$, where $\mathrm{k}$ is a positive integer.

The entire codeword is concatenated with unary part and binary part, and one bit is inserted between both for identification

\section{SIMULATION AND RESULTS}

Results of compression and decompression are compared in terms of compression ratio, Peak signal to noise ratio, Mean square error. The number of decompositions determines the quality of compressed image. The number of decompositions also determines the resolution of the lowest level in wavelet domain. If a larger number of decompositions is used, it will provide more success in resolving important DWT coefficients from less important coefficients. The HVS is less sensitive to removal of smaller details. At present, the most widely used objective distortion measures are the MSE and the related PSNR. They can easily be computed to represent the deviation of the distorted image from the original image in the pixel wise sense. However, in practical viewing situations, human beings are usually not concentrated on pixel differences alone, except for particular applications such as medical imaging, where pixel wise precision can be very important. The subjective perceptual quality includes surface smoothness, edge sharpness and continuity, proper background noise level, and so on. Image compression techniques induce various types of visual artifacts that affect the human viewing experience in many distinct ways, even if the MSE or PSNR level is adjusted to be about equal. It is generally agreed that MSE or PSNR does not correlate well with the visual quality perceived by human beings, since MSE is computed by adding the squared differences of individual pixels without considering the spatial interaction among adjacent pixels.

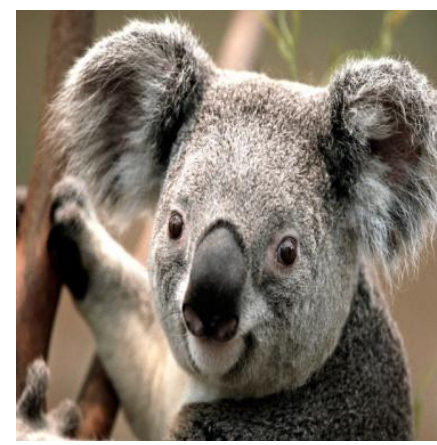

(a) Koala

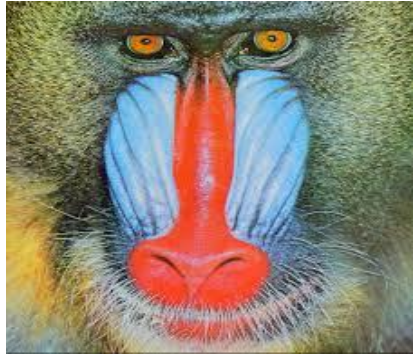

(b) Baboon 


\section{International Advanced Research Journal in Science, Engineering and Technology}

Vol. 2, Issue 12, December 2015

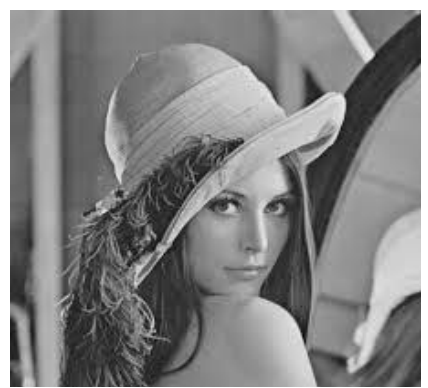

(c) Lena

Resulting decompressed images are:

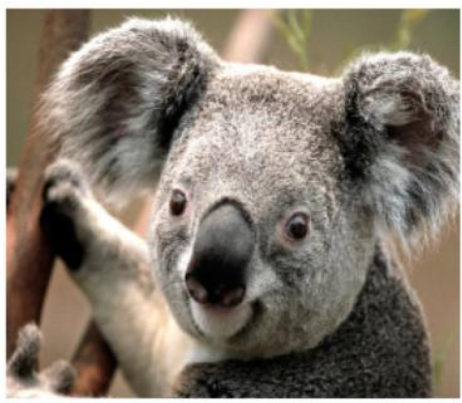

(a) Koala

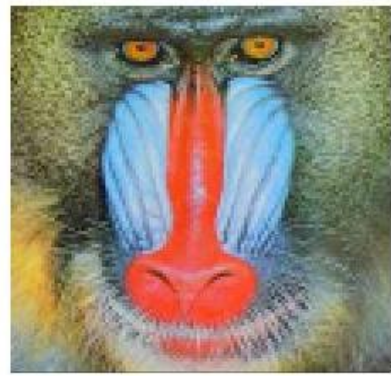

(b)Baboon

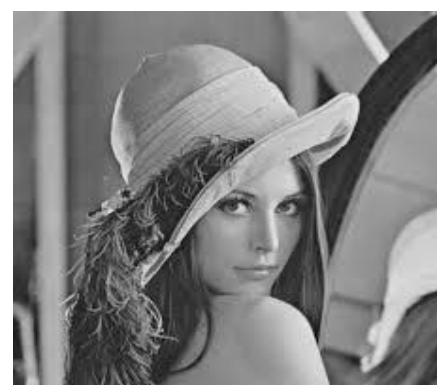

(c) Lena

\begin{tabular}{|l|l|l|l|l|l|}
\hline Figure & $\begin{array}{l}\text { Comp } \\
\text { ressio } \\
\text { n ratio }\end{array}$ & $\begin{array}{l}\text { Elapsed } \\
\text { time for } \\
\text { compressi } \\
\text { on (in } \\
\text { seconds) }\end{array}$ & $\begin{array}{l}\text { Mean } \\
\text { Square } \\
\text { Error }\end{array}$ & $\begin{array}{l}\text { PSNR } \\
\text { (in } \\
\text { dB) }\end{array}$ & $\begin{array}{l}\text { Elapsed } \\
\text { time for } \\
\text { Decompr } \\
\text { ession } \\
\text { (in } \\
\text { seconds) }\end{array}$ \\
\hline Koala & 4.8213 & 134.5657 & 25.34 & 34.126 & 17.390 \\
\hline Baboon & 5.0105 & 131.454 & 17.14 & 35.824 & 15.792 \\
\hline Lena & 6.560 & 124.948 & 253.67 & 24.122 & 13.240 \\
\hline
\end{tabular}

\section{CONCLUSION}

A new image compression scheme based on discrete wavelet transform is proposed in this research which provides sufficient high compression ratios with no appreciable degradation of image quality. The effectiveness and robustness of this approach has been justified using a set of real images. The wavelet transforms, together with embedded bit plane coding, provide a scalable representation of images such that

Different reconstruction qualities are obtained by truncating a single bit stream at different rates. Wavelets are better suited to time-limited data and wavelet based compression technique maintains better image quality by reducing errors. This result is better because compression technique make it best to transform image sub band Haar wavelet process. The Haar wavelet transform is the simplest one to implement, and it is the fastest. However, because of its discontinuity, it is not optimal to simulate a continuous signal.

\section{FUTURE OF IMAGE COMPRESSION:}

- Be able to compress large images

- Use single decompression architecture

- Transmission in noisy environments

- Robustness to bit-errors

- Progressive transmission by Robustness to bit-errors

- Progressive transmission by pixel accuracy and resolution

\section{REFERENCES}

[1]. Bhonde Nilesh, Shinde Sachin, Nagmode Pradip, D.B. Rane, "Image Compression Using Discrete Wavelet Transform”, International Journal of Computer Technology and Electronics Engineering (IJCTEE) MarchApril 2013

[2]. Cung Nguyen, Member, IEEE, and G. Robert Redinbo, Fellow, IEEE, "Fault Tolerance Design in JPEG 2000 Image Compression System", IEEE TRANSACTIONS ON DEPENDABLE AND SECURE COMPUTING, March 2005

[3]. Dharmendra Kumar Gangwar, Bhagwat University, Ajmer, “ Compression and Development analysis on an image using Haar wavelet transform", International Journal of Advanced Technology \& Engineering Research (IJATER)

[4]. Zunera Idrees, Eliza, Linnaeus University, "Image Compression by Using Haar Wavelet Transform and Singular Value Decomposition".

[5]. G.K.Wallace, "The JPEG still compression standard".Commun.ACM,Apr.1991.

[6] T.Acharya and Ping-Sing Tsai, JPEG2000 Standard for Image Compression Concepts, algorithms and VLSI Architectures. John Wiley \& Sons press, 2005.

[7] R. C. Gonzalez and R. E. Woods, "Digital Image Processing", Addison Wesley, 2004

[8] V.Ratnakar and M.Livny. RD-OPT: An efficient algorithm for optimizing DCT quantization tables. Data Compression Conference.

[9] A.Skodras, Christopoulos and T Ebrahimi. The JPEG 2000 still image compression standard. IEEE Signal processing magazine

[10] Sonja Grgic, Kresimir Kers, Mislav Grgic, "Image Compression Using Wavelets" IEEE, ISIE 99Bled Slovenia.

[11] Sunil Kore and Akshay Bhosale, "An Efficient Image Compression Technique using 2D-DWT and FELICS Algorithm for Different Class of Images (COI)", IJCEM International Journal of Computational Engineering \& Management,2012

[12] JPEG 2000 Part 1 Final Draft International Standard, ISO/IEC FDIS15444-1, Dec. 2000.

[13] P. G. Howard and J. S. Vitter, "Fast and efficient lossless image compression," in Proc. IEEE Int. Conf. Data Compression, 1993, pp. 501-510.

[14] Yi-Qiang Hu, Hung-Hseng Hsu and Bing-FeiWuAn, "Integrated Method to Image Compression Using the Discrete Wavelet Transform" IEEE International Symposium on Circuits and Systems,June 9-12, 1997, Hong Kong.

[15] Tsung-Han Tsai, Yu-Hsuan Lee, and Yu-Yu Lee, "Design and Analysis of High-Throughput Lossless Image Compression Engine using VLSIOriented FELICS Algorithm" IEEE trans. on VLSI Systems, Vol. 18, No.1, January 2010. 